\title{
INTRODUCTION TO QUANTUM LIE ALGEBRAS
}

\author{
GUSTAV W. DELIUS \\ Department of Physics, University of Bielefeld \\ Postfach 1001 31, 33501 Bielefeld, Germany \\ E-mail: delius@mth.kcl.ac.uk \\ http:/www.mth.kcl.ac.uk/ delius
}

\begin{abstract}
Quantum Lie algebras are generalizations of Lie algebras whose structure constants are power series in $h$. They are derived from the quantized enveloping algebras $U_{h}(\mathfrak{g})$. The quantum Lie bracket satisfies a generalization of antisymmetry. Representations of quantum Lie algebras are defined in terms of a generalized commutator.

The recent general results about quantum Lie algebras are introduced with the help of the explicit example of $\left(\mathfrak{s l}_{2}\right)_{h}$.
\end{abstract}

1. Introduction. The subject of this paper are the question marks in the following diagram

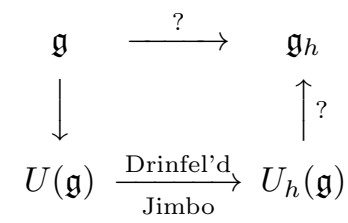

Drinfel'd [1] and Jimbo [2] have shown how to define a quantization $U_{h}(\mathfrak{g})$ of the enveloping algebra $U(\mathfrak{g})$ of any simple complex Lie algebra $\mathfrak{g}$. These quantized enveloping algebras have proven to be important in several branches of mathematics and physics and have been studied in detail. In contrast, very little is know about the quantization of the Lie algebras themselves.

The approach to the quantization of Lie algebras which was initiated in $[3,8]$ consists of making use of the known quantization of the corresponding enveloping algebras. The quantum Lie algebras are extracted from the quantized enveloping algebras in the same way as the unquantized Lie algebras can be extracted from the unquantized enveloping algebras.

1991 Mathematics Subject Classification: 81R50.

The paper is in final form and no version of it will be published elsewhere. 
In an alternative approach, initiated by Woronowicz [5], one can extract quantum Lie algebras from the formalism of bicovariant differential calculi on quantum groups. This approach always leads to quantum Lie algebras which have a larger dimension than their classical counterpart. We now know that these algebras are not simple and that our quantum Lie algebras are simple subalgebras of these. Here we will deal only with the simple quantum Lie algebras obtained from our algebraic approach. For works on the Woronowicz algebras see e.g. [6]. For an approach specific to quantum $\mathfrak{s l}_{n}$ but related to our general approach see [9].

In this paper we will use the simplest example, namely the three-dimensional Lie algebra $\mathfrak{s l}_{2}$ so familiar to physicists, to introduce the general results about quantum Lie algebras which have recently been obtained. We will also give a matrix representation of this algebra which has not yet been published.

2. The Lie algebra $\mathfrak{s l}_{2}$. Complex Lie algebras $\mathfrak{g}$ in general are vector spaces over $\mathfrak{C}$ equipped with a non-associative product, commonly denoted as the Lie bracket. This is a linear map [,] $: \mathfrak{g} \otimes \mathfrak{g} \rightarrow \mathfrak{g}$ which satisfies

$$
\begin{aligned}
{[a, b] } & =-[b, a] & & \text { antisymmetry } \\
{[a,[b, c]] } & =[[a, b], c]+[b,[a, c]] & & \text { Jacobi identity. }
\end{aligned}
$$

The complex simple Lie algebra $\mathfrak{s l}_{2}$ is spanned as a vector space by three elements $X^{+}, X^{-}$ and $H$. The Lie bracket is given by

$$
\left[X^{+}, X^{-}\right]=H, \quad\left[H, X^{ \pm}\right]= \pm 2 X^{ \pm} .
$$

Together with the antisymmetry property and the bilinearity these three relations define the Lie bracket on the whole algebra uniquely.

3. The enveloping algebra $U\left(\mathfrak{s l}_{2}\right)$. The enveloping algebra $U\left(\mathfrak{s l}_{2}\right)$ is the associative unital algebra over $\mathfrak{C}$ generated by the three generators $X^{+}, X^{-}$and $H$ and the commutation relations

$$
X^{+} X^{-}-X^{-} X^{+}=H, \quad H X^{ \pm}-X^{ \pm} H= \pm 2 X^{ \pm} .
$$

In other words: $U\left(\mathfrak{s l}_{2}\right)$ contains all possible ordered polynomials in the three generators but two such polynomials are equal if they are related by the above commutation relations. It can be seen that the relations allow one to commute all $X^{-}$to the left and all $X^{+}$ to the right. Thus as a basis for $U\left(\mathfrak{s l}_{2}\right)$ one can choose $\left\{\left(X^{-}\right)^{n} H^{m}\left(X^{+}\right)^{l} \mid n, m, l \in \mathfrak{N}\right\}$, known as the Poincaré-Birkhoff-Witt basis.

The enveloping algebra $U\left(\mathfrak{s l}_{2}\right)$ is clearly infinite dimensional. It contains $\mathfrak{s l}_{2}$ as the subspace spanned by $X^{-}, X^{+}$and $H$. This subspace is closed under the commutator and the commutator coincides with the Lie bracket as defined in (2.3). Because of this the mind of a physicist tends not to distinguish between Lie brackets and commutators. Below however it will be crucial to keep the two concepts clearly separated.

The enveloping algebra is a Hopf algebra. In this paper we will only need to know that this implies that one can define an action of the enveloping algebra on itself, the so 
called adjoint action. For $U\left(\mathfrak{s l}_{2}\right)$ it is defined by

$$
\left(\operatorname{ad} X^{ \pm}\right) a=X^{ \pm} a-a X^{ \pm}, \quad(\operatorname{ad} H) a=H a-a H, \quad \forall a \in U\left(\mathfrak{s l}_{2}\right) .
$$

Thus for the generators the adjoint action is just the commutator. For products of generators the adjoint action is obtained from the above by the defining property of an action, i.e., $(\operatorname{ad} a b)=(\operatorname{ad} a)(\operatorname{ad} b)$.

4. The quantized enveloping algebra $U_{h}\left(\mathfrak{s l}_{2}\right)$. The quantized enveloping algebra $U_{h}\left(\mathfrak{s l}_{2}\right)[1,2,7]$ too is an associative unital algebra generated by the three generators $X^{+}, X^{-}$and $H$. However it is an algebra over $\mathfrak{C}[[h]]$, the ring of formal power series in an indeterminate $h$ (which in physical applications may be related to Planck's constant, but not necessarily linearly so). The commutation relations are deformed with respect to (3.1). They now read

$$
X^{+} X^{-}-X^{-} X^{+}=\frac{q^{H}-q^{-H}}{q-q^{-1}}, \quad H X^{ \pm}-X^{ \pm} H= \pm 2 X^{ \pm},
$$

where $q=e^{h}$. Thus the commutator of $X^{+}$and $X^{-}$gives an infinite power series in $\mathrm{h}$. The first term in the series is just $H$, as classically, but the higher order terms in $h$ (the quantum corrections) are non-linear in $H$.

The important property of the deformation (4.1) is that it still defines a Hopf algebra. However also the Hopf algebra structure is deformed and this leads in particular to a deformed adjoint action

$$
\begin{aligned}
\left(\operatorname{ad} X^{ \pm}\right) a & =X^{ \pm} a q^{H / 2}-q^{\mp 1} q^{H / 2} a X^{ \pm}, & \\
(\operatorname{ad} H) a & =H a-a H, & \forall a \in U\left(\mathfrak{s l}_{2}\right) .
\end{aligned}
$$

5. A quantum Lie algebra $\mathfrak{L}_{h}\left(\mathfrak{s l}_{2}\right)$ inside $U_{h}\left(\mathfrak{s l}_{2}\right)$. As explained in Section 3 the Lie algebra $\mathfrak{s l}_{2}$ can be viewed as a subspace of the enveloping algebra $U\left(\mathfrak{s l}_{2}\right)$ which is spanned by the generators $X^{+}, X^{-}$and $H$ and on which the Lie bracket is given by the commutator. We would now like to obtain the quantum Lie algebra $\mathfrak{L}_{h}\left(\mathfrak{s l}_{2}\right)$ in a similar manner from the quantized enveloping algebra $U_{q}\left(\mathfrak{s l}_{2}\right)$.

However, in the quantum case the space ${ }^{1}$ spanned by the generators $X^{+}, X^{-}$and $H$ does not close under the commutator. The non-linear terms in $\mathrm{H}$ in the commutation relations (4.1) create a problem. The first idea is to replace the role of the commutator by the adjoint action. As we had seen the two coincide in the classical case but differ in the quantum case. If we find a three-dimensional subspace of $U_{q}\left(\mathfrak{s l}_{2}\right)$ which is closed under the adjoint action then we can define a quantum Lie bracket on this space by

$$
[a, b]_{h} \equiv(\operatorname{ad} a) b .
$$

The space spanned by

$$
X_{h}^{ \pm}=\sqrt{\frac{2}{q+q^{-1}}} q^{-H / 2} X^{ \pm}, \quad H_{h}=\frac{2}{q+q^{-1}}\left(q X^{+} X^{-}-q^{-1} X^{-} X^{+}\right),
$$

\footnotetext{
${ }^{1}$ To be pedantic, because $\mathfrak{C}[[h]]$ is a ring and not a field, we should not speak of vector spaces but rather of $\mathfrak{C}[[h]]$-modules. However in this paper we would prefer not to dwell on such subtleties.
} 
satisfies this requirement. Indeed, using (4.2), one can calculate the adjoint action of these elements on each other. The reader is urged to perform these calculations. He will find

$$
\begin{aligned}
{\left[X_{h}^{+}, X_{h}^{-}\right]_{h} } & =H_{h}, & {\left[X_{h}^{-}, X_{h}^{+}\right]_{h} } & =-H_{h}, \\
{\left[H_{h}, X_{h}^{ \pm}\right]_{h} } & = \pm 2 q^{ \pm 1} X_{h}^{ \pm}, & & {\left[X_{h}^{ \pm}, H_{h}\right]_{h}=\mp 2 q^{\mp 1} X_{h}^{ \pm} } \\
{\left[H_{h}, H_{h}\right]_{h} } & =2\left(q-q^{-1}\right) H_{h}, & {\left[X_{h}^{ \pm}, X_{h}^{ \pm}\right]_{h} } & =0 .
\end{aligned}
$$

These quantum Lie bracket relations are the quantum analog of the Lie bracket relations (2.3). To zeroth order in $h$ they agree with the classical Lie bracket relations.

6. $q$-antisymmetry. We stress that the quantum Lie algebra $\mathfrak{L}_{h}\left(\mathfrak{s l}_{2}\right)$ is not a Lie algebra. The quantum Lie bracket defined by (5.3) does not satisfy the properties of antisymmetry (2.1) and Jacobi (2.2). Instead it satisfies an interesting generalization of antisymmetry which involves the operation $q \rightarrow 1 / q$. The details are as follows:

We define $q$-conjugation $\sim: \mathfrak{C}[[h]] \rightarrow \mathfrak{C}[[h]]$ as the $\mathfrak{C}$-linear ring automorphism defined by $\tilde{h}=-h$ (and thus $\tilde{q}=1 / q$ ). We extend this to a $q$-conjugation on $\mathfrak{L}_{h}\left(\mathfrak{s l}_{2}\right)$ by defining

$$
\left(a X_{h}^{+}+b X_{h}^{-}+c H_{h}\right)^{\sim}=\tilde{a} X_{h}^{+}+\tilde{b} X_{h}^{-}+\tilde{c} H_{h} .
$$

Then the quantum Lie bracket satisfies

$$
[x, y]_{h}=-[\tilde{y}, \tilde{x}] \quad \forall x, y \in \mathfrak{L}_{h}\left(\mathfrak{s l}_{2}\right) .
$$

We call this property $q$-antisymmetry. This property can easily be verified for the quantum Lie bracket relations (5.3) even though it is not at all evident from the definition (5.1) of the quantum Lie bracket. As shown in [4] all quantum Lie algebras (defined below) possess this $q$-antisymmetry.

We have not yet discovered the $q$-analog of the Jacobi identity.

7. General definition of $\mathfrak{L}_{h}(\mathfrak{g})$. The quantum Lie algebra $\mathfrak{L}_{h}\left(\mathfrak{s l}_{2}\right)$ constructed in Section 5 is the simplest example of the following general definition.

Definition 1. A quantum Lie algebra $\mathfrak{L}_{h}(\mathfrak{g})$ inside $U_{h}(\mathfrak{g})$ is a finite-dimensional indecomposable ad - submodule of $U_{h}(\mathfrak{g})$ endowed with the quantum Lie bracket $[a, b]_{h}=$ $(\operatorname{ad} a) b$ such that

1. $\mathfrak{L}_{h}(\mathfrak{g})$ is a deformation of $\mathfrak{g}$, i.e., $\mathfrak{L}_{h}(\mathfrak{g})=\mathfrak{g}(\bmod h)$.

2. $\mathfrak{L}_{h}(\mathfrak{g})$ is invariant under the $q$-Cartan involution $\tilde{\theta}$, the $q$-antipode $\tilde{S}$ and any diagram automorphism $\tau$ of $U_{q}(\mathfrak{g})$.

Property 2 plays an important role in the investigations into the general structure of quantum Lie algebras in [3]. In particular it allows the definition of a quantum Killing form. We refer the reader to the paper [3] for more information on these matters. It is shown in [3] that given any module satisfying all properties of the definition except property 2 one can always construct from it a quantum Lie algebra $\mathfrak{L}_{h}(\mathfrak{g})$ which satisfies property 2 as well. Thus this extra requirement is not too strong.

While finding a quantum Lie algebra $\mathfrak{L}_{h}\left(\mathfrak{s l}_{2}\right)$ inside $U_{h}\left(\mathfrak{s l}_{2}\right)$ was easy, performing the similar task for other groups is much more involved. However, as reported in [3], it has 
been done by using the computer algebra program Mathematica for the Lie algebras $\mathfrak{g}=\mathfrak{s l}_{3}, \mathfrak{s l}_{4}, \mathfrak{s o}_{5}=\mathfrak{s p}_{4}$ and $G_{2}$. There is also a method for constructing quantum Lie algebras in general using the universal R-matrix. This method has been applied in [8] to obtain quantum Lie algebras for $\mathfrak{g}=\mathfrak{s l}_{n}$ for all $n$. The method is described also in [4]. This construction proves in particular that quantum Lie algebras exist for all $\mathfrak{g}$. However the determination of the structure of these quantum Lie algebras has not yet been performed except in the above mentioned cases.

8. The abstract quantum Lie algebra $\left(\mathfrak{s l}_{2}\right)_{h}$. The space spanned by the three generators $X_{h}^{+}, X_{h}^{-}$and $H_{h}$ given in (5.2) is not the only three dimensional subspace of $U_{h}\left(\mathfrak{s l}_{2}\right)$ which is closed under the adjoint action and gives rise to a quantum Lie algebra $\mathfrak{L}_{h}\left(\mathfrak{s l}_{2}\right)$. But it is easy to convince oneself of the fact that any quantum Lie algebra $\mathfrak{L}_{h}\left(\mathfrak{s l}_{2}\right)$ inside $U_{h}\left(\mathfrak{s l}_{2}\right)$ is spanned by three elements of the form

$$
X_{h}^{ \pm}=\sqrt{\frac{2}{q+q^{-1}}} q^{-H / 2} X^{ \pm} \mathcal{P}(C), \quad H_{h}=\frac{2}{q+q^{-1}}\left(q X^{+} X^{-}-q^{-1} X^{-} X^{+}\right) \mathcal{P}(C),
$$

where $\mathcal{P}(C)$ can be any polynomial in the Casimir element

$$
C=\frac{1}{\left(q^{3}+q^{-3}\right)}\left(\left(q-q^{-1}\right)^{2} X^{+} X^{-}+q^{H-1}+q^{-H+1}\right)
$$

whose coefficients sum to 1 . Because of the properties of the Casimir element, all these quantum Lie algebras lead to the same quantum Lie bracket relations (5.3). Thus all quantum Lie algebras $\mathfrak{L}_{h}\left(\mathfrak{s l}_{2}\right)$ are isomorphic as algebras. This defines an abstract quantum Lie algebra $\left(\mathfrak{s l}_{2}\right)_{h} \cdot\left(\mathfrak{s l}_{2}\right)_{h}$ is the algebra spanned by three abstract generators $X_{h}^{+}, X_{h}^{-}$ and $H_{h}$ with the Lie bracket relations (5.3). The concrete quantum Lie algebras $\mathfrak{L}_{h}\left(\mathfrak{s l}_{2}\right)$ are just different embeddings of $\left(\mathfrak{s l}_{2}\right)_{h}$ into $U_{h}\left(\mathfrak{s l}_{2}\right)$.

Similarly, as proven in [4], there is a unique abstract quantum Lie algebra $\mathfrak{g}_{h}$ for any simple complex Lie algebra $\mathfrak{g}$.

THEOREM 1. All concrete quantum Lie algebras $\mathfrak{L}_{h}(\mathfrak{g})$ for the same $\mathfrak{g}$ are isomorphic to a unique abstract quantum Lie algebra $\mathfrak{g}_{h}$.

Furthermore it has been shown that the structure constants of $\mathfrak{g}_{h}$ are equal to the $q$-Clebsch-Gordon coefficients for adjoint×adjoint into adjoint.

9. Representations of $\left(\mathfrak{s l}_{2}\right)_{h}$. An $n$-dimensional representation of a Lie algebra is a linear map $\pi$ from the Lie algebra into the $n \times n$ matrices, $\pi: \mathfrak{g} \rightarrow \operatorname{Mat}_{n}(\mathfrak{C})$, such that the Lie bracket is realized as the matrix commutator, i.e.,

$$
\pi([a, b])=\pi(a) \pi(b)-\pi(b) \pi(a) .
$$

Such maps $\pi$ exist because the commutator also possesses the defining properties of the Lie bracket, namely antisymmetry (2.1) and Jacobi (2.2).

To arrive at a good definition of a representation of a quantum Lie algebra we have to find a $q$-generalization of the commutator which is $q$-antisymmetric in the sense of (6.2). We propose the following definition:

Definition 2. An $n$-dimensional representation of a quantum Lie algebra $\mathfrak{g}_{h}$ consists of 
- a linear map $\pi: \mathfrak{g}_{h} \rightarrow \operatorname{Mat}_{n}(h)$,

- a $q$-conjugation $\sim: \operatorname{Mat}_{n}(\mathfrak{C}[[h]]) \rightarrow \operatorname{Mat}_{n}(\mathfrak{C}[[h]])$,

such that

$$
\begin{aligned}
\pi(\tilde{a}) & =\tilde{\pi}(a), \\
\pi\left([a, b]_{h}\right) & =\pi(a) \pi(b)-(\tilde{\pi}(b) \tilde{\pi}(a))^{\sim} .
\end{aligned}
$$

By definition [3] a $q$-conjugation is $q$-linear, i.e., $(\lambda a)^{\sim}=\tilde{\lambda} \tilde{a} \forall \lambda \in \mathfrak{C}[[h]], a \in$ $\operatorname{Mat}_{n}(\mathfrak{C}[[h]])$, and is an involution, i.e., $\tilde{\tilde{a}}=a \forall a \in \operatorname{Mat}_{n}(\mathfrak{C}[[h]])$.

To illustrate this definition we will give the 2-dimensional representation of $\left(\mathfrak{s l}_{2}\right)_{h}$. The representation matrices are

$$
\pi\left(X_{h}^{+}\right)=\sqrt{\frac{q+q^{-1}}{2}}\left(\begin{array}{ll}
0 & 1 \\
0 & 0
\end{array}\right), \quad \pi\left(H_{h}\right)=\left(\begin{array}{cc}
q & 0 \\
0 & -q^{-1}
\end{array}\right), \quad \pi\left(X_{h}^{-}\right)=\pi\left(X_{h}^{+}\right)^{t}
$$

and the $q$-conjugation is given by

$$
\begin{array}{ll}
\left(\begin{array}{ll}
1 & 0 \\
0 & 0
\end{array}\right)^{\sim}=\frac{1}{q+q^{-1}}\left(\begin{array}{cc}
2 q & 0 \\
0 & q-q^{-1}
\end{array}\right), & \left(\begin{array}{ll}
0 & 1 \\
0 & 0
\end{array}\right)^{\sim}=\left(\begin{array}{ll}
0 & 1 \\
0 & 0
\end{array}\right), \\
\left(\begin{array}{ll}
0 & 0 \\
0 & 1
\end{array}\right)^{\sim}=\frac{1}{q+q^{-1}}\left(\begin{array}{cc}
q^{-1}-q & 0 \\
0 & 2 q^{-1}
\end{array}\right), & \left(\begin{array}{ll}
0 & 0 \\
1 & 0
\end{array}\right)^{\sim}=\left(\begin{array}{ll}
0 & 0 \\
1 & 0
\end{array}\right) .
\end{array}
$$

The reader is urged to check that the $q$-commutators of the representation matrices in (9.5) do indeed reproduce the algebra (5.3).

The author has a construction for representations of $\left(\mathfrak{s l}_{2}\right)_{h}$ of any dimension.

10. Discussion. We have reviewed quantum Lie algebras by using the explicit example of $\mathfrak{s l}_{2}$. Quantum Lie algebras were originally defined as certain subspaces $\mathfrak{L}_{h}(\mathfrak{g})$ of the quantized enveloping algebras $U_{h}(\mathfrak{g})$. It was then found that as algebras all the $\mathfrak{L}_{h}(\mathfrak{g})$ are isomorphic to an abstract quantum Lie algebra $\mathfrak{g}_{h}$. We have seen that $q$-conjugation $q \rightarrow 1 / q$ plays a central role in the theory of quantum Lie algebras. In particular, the quantum Lie bracket turns out to be $q$-antisymmetric in the sense of (6.2). This has lead to the definition (9.3) of a $q$-commutator to represent the quantum Lie bracket.

Many definitions for $q$-commutators can be found in the literature. They generally differ from the usual commutator by multipying the terms by certain powers of $q$. Our definition (9.3) of the $q$-commutator is quite different and arises naturally in the theory of quantum Lie algebras. It will be interesting to study its physical applications.

Drinfel'd has introduced a quantized enveloping algebra $U_{h}(\mathfrak{g})$ for any complex simple Lie algebra $\mathfrak{g}$. There is no definition of what a quantized enveloping algebra is in general. Similarly we have so far defined the concept of a quantum Lie algebra $\mathfrak{g}_{h}$ only for complex simple (finite-dimensional) Lie algebras $\mathfrak{g}$. There are however indications that an axiomatic definition of quantum Lie algebras can be obtained which parallels the axiomatic definition of Lie algebras through the properties of antisymmetry (2.1) and Jacobi (2.2).

Antisymmetry and Jacobi identity are the necessary and sufficient conditions for an algebra to have a representation in terms of commutators. Similarly $q$-antisymmetry 
and $q$-Jacobi should be the necessary and sufficient conditions for an algebra to have a representation in terms of $q$-commutators. The $q$-antisymmetry (6.2) is clearly a necessary condition but we are still searching for the $q$-Jacobi identity which gives a sufficient condition. It could be hoped that this will then also finally lead to an axiomatic definition of quantized enveloping algebras. For up to date information see [10].

\section{References}

[1] V. G. Drinfel'd, Hopf algebras and the quantum Yang-Baxter equation, Sov. Math. Dokl. 32 (1985) 254.

[2] M. Jimbo, A q-Difference Analogue of $U(g)$ and the Yang-Baxter Equation, Lett. Math. Phys. 10 (1985) 63.

[3] G. W. Delius, A. Hüffmann, On Quantum Lie Algebras and Quantum Root Systems, q-alg/9506017, J. Phys. A. 29 (1996) 1703.

[4] G. W. Delius, M. D. Gould, Quantum Lie Algebras, their existence, uniqueness and qantisymmetry, KCL-TH-96-05, q-alg/9605025, Commun. Math. Phys. (in print).

[5] S. L. Woronowicz, Differential Calculus on Compact Matrix Pseudogroups (Quantum Groups), Comm. Math. Phys. 122 (1989) 125.

[6] P. Aschieri, L. Castellani, An introduction to noncommutative differential geometry on quantum groups, Int. J. Mod. Phys. A8 (1993) 1667;

D. Bernard, Quantum Lie Algebras and Differential Calculus on Quantum Groups, Prog. Theo. Phys. Suppl. 102 (1990) 49;

B. Jurco, Differential Calculus on Quantized Simple Lie Groups, Lett. Math. Phys. 22 (1991) 177 ;

P. Schupp, P. Watts, B. Zumino, Bicovariant Quantum Algebras and Quantum Lie Algebras, Commun. Math. Phys. 157 (1993) 305;

P. Schupp, Quantum Groups, Non-Commutative Differential Geometry and Applications, hep-th/9312075 (1993);

K. Schmüdgen, A. Schüler, Classification of Bicovariant Differential Calculi on Quantum Groups of Type A, B, C and D, Comm. Math. Phys.107 (1995) 635;

A. Sudbery, Quantum Lie Algebras of Type $A_{n}$, q-alg/9510004.

[7] V. Chari, A. Pressley, A Guide to Quantum Groups, Cambridge University Press (1994).

[8] G.W. Delius, M.D. Gould, A. Hüffmann, Y.-Z. Zhang, Quantum Lie algebras associated to $U_{q}\left(g l_{n}\right)$ and $U_{q}\left(s l_{n}\right)$, q-alg/9508013.

[9] V. Lyubashenko and A. Sudbery, Quantum Lie algebras of type $A(N)$, q-alg/9510004.

[10] World Wide Web, http:/www.mth.kcl.ac.uk/ delius/q-lie.html. 